\title{
Contact Analysis of a Dry Friction Clutch System
}

\author{
Oday I. Abdullah and Josef Schlattmann \\ Department of System Technology and Mechanical Design Methodology, Hamburg University of Technology, Denickestraße 17, \\ Gebäude L, 21073 Hamburg, Germany \\ Correspondence should be addressed to Oday I. Abdullah; oday.abdullah@tu-harburg.de
}

Received 9 June 2013; Accepted 22 July 2013

Academic Editors: J. Hu, K. Ismail, K. Mekheimer, and K. T. Ooi

Copyright (C) 2013 O. I. Abdullah and J. Schlattmann. This is an open access article distributed under the Creative Commons Attribution License, which permits unrestricted use, distribution, and reproduction in any medium, provided the original work is properly cited.

\begin{abstract}
The numerical simulation of the friction clutch system (pressure plate, clutch disc, and flywheel) during the full engagement period (assuming no slipping between contact surfaces) is carried out using finite element method. Two types of load condition considered affect on the clutch elements during the full engagement period are the contact pressure of diaphragm spring and the centrifugal force. The study of the pressure distribution between the contact surfaces and the factors affecting it is one of the fundamentals in the process of designing the friction clutch to obtain accurate estimation of the temperature distribution during the slipping period and the contact stresses during the full engagement period. The investigation covers the effect of the contact stiffness factor FKN on the pressure distribution between contact surfaces, stresses, and penetration. The penalty and augmented Lagrange algorithms have been used to obtain the pressure distribution between contact surfaces. ANSYS13 software has been used to perform the numerical calculation in this paper.
\end{abstract}

\section{Introduction}

A clutch is a very important machine element which plays a main role in the transmission of power (and eventually motion) from one component (the driving part of the machine) to another (the driven part). A common and wellknown application for the clutch is in automotive vehicles where it is used to connect the engine and the gearbox. Furthermore, the clutch is used also extensively in production machinery of all types. When the clutch disc begins to engage, the contact pressure between the contact surfaces will increase to the maximum value at the end of the slipping period and will stay steady during the full engagement period. At high relative sliding velocity, high quantity of frictional heat is generated which leads to high temperature rise on the clutch disc surfaces and hence thermomechanical problems such as thermal deformations and thermoelastic instability can occur. This in turn can lead to thermal cracking and high rate of wear. The pressure distribution is essential factor effect on the performance of the friction clutch because of the heat generated between contact surfaces during the slipping period dependent on the pressure distribution.
Al-Shabibi and Barber [1] used the finite element method to find the transient solution of the temperature field and contact pressure distribution between two sliding disks. Twodimensional axisymmetric FE model used to explore an alternative method based on an eigenfunction expansion and a particular solution that can be used to solve the thermoelastic contact problem with frictional heating. Both constant speed and varying sliding speed are considered in this analysis. Results of the direct finite element simulation have been obtained using the commercial package ABAQUS. The results from the approximate solution show a good agreement with the results from the direct finite element simulation.

Choon et al. [2] used finite element method to study the effect of thermomechanical loads on the pressure plate and the hub plate of the friction clutch system. Three types of loads take into consideration are the thermal load due to the slipping occurs at the beginning of engagement, the contact pressure of diaphragm spring and the centrifugal force due to the rotation. Two- and three-dimensional finite element models were performed to obtain the temperature distributions and the stresses. The results show the significant 
TABLE 1: The properties of materials and operations.

\begin{tabular}{|c|c|}
\hline Parameters & Values \\
\hline Inner radius of friction material and axial cushion, $r_{i}(\mathrm{~m})$ & 0.06298 \\
\hline Outer radius of friction material and axial cushion, $r_{o}(\mathrm{~m})$ & 0.08721 \\
\hline Thickness of friction material (m), $t_{l}$ & 0.003 \\
\hline Thickness of the axial cushion (m), $t_{a x i}$ & 0.0015 \\
\hline Inner radius of pressure plate $(\mathrm{m}), r_{i p}$ & 0.05814 \\
\hline Outer radius of pressure plate $(\mathrm{m}), r_{o p}$ & 0.09205 \\
\hline Thickness of the pressure plate $(\mathrm{m}), t_{p}$ & 0.00969 \\
\hline Inner radius of flywheel $(\mathrm{m}), r_{i f}$ & 0.04845 \\
\hline Outer radius of flywheel $(\mathrm{m}), r_{o f}$ & 0.0969 \\
\hline Thickness of the flywheel $(\mathrm{m}), t_{f}$ & 0.01938 \\
\hline Pressure, $p(\mathrm{MPa})$ & 1 \\
\hline Coefficient of friction, $\mu$ & 0.2 \\
\hline Number of friction surfaces, $n$ & 2 \\
\hline Torque (Nm), $T$ & 432 \\
\hline Maximum angular slipping speed, $\omega_{o}(\mathrm{rad} / \mathrm{sec})$ & 200 \\
\hline Young's modulus for friction material, $E_{l}(\mathrm{GPa})$ & 0.30 \\
\hline Young's modulus for pressure plate, flywheel, and axial cushion $\left(E_{p}, E_{f}\right.$, and $\left.E_{a x i}\right),(\mathrm{Gpa})$ & 125 \\
\hline Poisson's ratio for friction material & 0.25 \\
\hline Poisson's ratio for pressure plate, flywheel, and axial cushion & 0.25 \\
\hline Density for friction material, $\left(\mathrm{kg} / \mathrm{m}^{3}\right), \rho_{l}$ & 2000 \\
\hline Density for pressure plate, flywheel, and axial cushion, $\left(\mathrm{kg} / \mathrm{m}^{3}\right),\left(\rho_{p}, \rho_{f}\right.$, and $\left.\rho_{\text {axi }}\right)$ & 7800 \\
\hline
\end{tabular}

effect of the thermal load on the temperatures and stresses; therefore, it is desirable to increase the thickness of the pressure plate as much as possible to increase the thermal capacity of the pressure plate to reduce the thermal stresses. High stress intensity value occurs around the fillet region of the window in the hub plate.

Shahzamanian et al. [3] used numerical simulation to study the transient and contact analysis of functionally graded (FG) brake disk. The material properties vary in the radial direction from full metal at the inner radius to that of full ceramic at the outer radius. The coulomb contact friction is considered between the pad and the brake disk. Two-dimensional finite element model was used in the work to obtain the pressure distribution, total stresses, pad penetration, friction stresses, heat flux, and temperature during the contact using different values of the contact stiffness factor. It was found that the contact pressure and contact total stress increase when the contact stiffness factor increases and the gradation of the metal ceramic has significant effect on the thermomechanical response of FG brake disks. Also, it can be concluded that when the thickness of the pad increases, the contact status between pad and disc changes from sticking to contact and then to near contact.

El-Sherbiny and Newcomb [4] used the finite difference method to setup equations to express the heat balance at every region in the clutch. They determined the temperatures at various elements when band contact occurs between the rubbing surfaces during the operation of an automotive clutch. Temperatures distributions are determined for contact area of a different band width on the both clutch facing. Both single and repeated engagements made at regular interval are considered.

Al-Bahkali and Barber [5] developed a two-dimensional finite element model to determine the nonlinear steady-state configuration of a two-dimensional thermoelastic system involving sliding in the plane with frictional heat generation. Above a certain critical speed, this causes the uniform pressure solution to be unstable and the final steady-state configuration involves regions of separation at the interface and associated temperature and displacement fields that migrate over the contacting bodies in the direction of sliding. Also, they presented new algorithm to determine this migration speed by iteration to be able to use a reference frame in which the fields are stationary.

Yevtushenko et al. [6] applied one-dimensional transient heat conductivity to study the contact problem of a sliding of two semispaces, which induces effects of friction, heat generation, and water during braking. In the present temperature analysis the capacity of the frictional source on the contact plane dependens on the time of braking. The problem The problem solved exactly using the Laplace transform technique. Numerical results for the temperature are obtained for the different values of the input parameter, which characterize the duration of the increase of the contact pressure during braking from zero to the maximum value. An analytical formula for the abrasive wear of the contact plane is obtained on the assumption that the wear coefficient is the linear function of the contact temperature.

In this paper the finite element method was used to study the contact pressure, stresses, and penetration during the full 


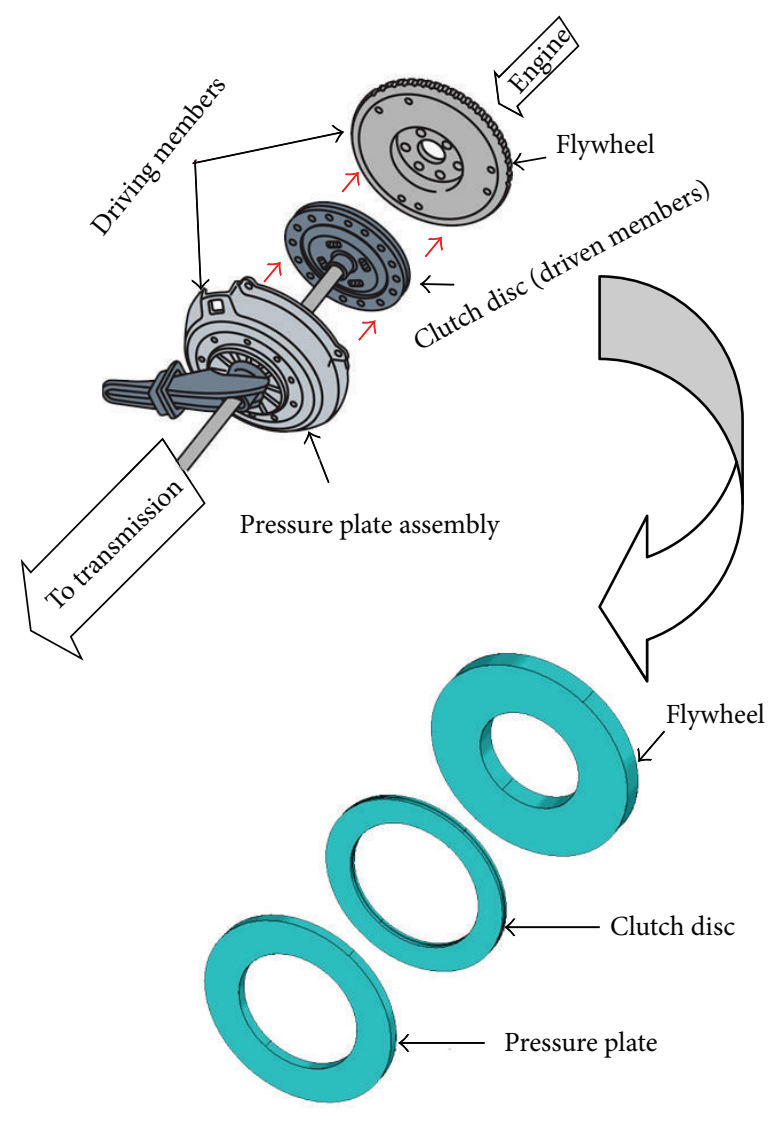

FIGURE 1: The main parts of clutch system.

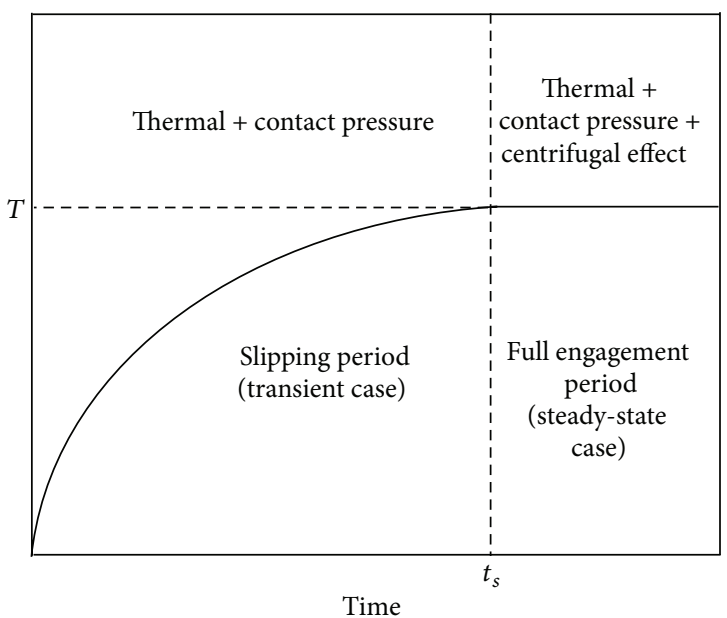

FIGURE 2: The load conditions during the engagement cycle of the friction clutch.

engagement period of the clutches using the penalty and augmented Lagrange contact algorithms. Moreover, sensitivity study for the contact pressure and penetration is presented to indicate the importance of the contact stiffness between contact surfaces of the friction clutch elements (flywheel, clutch disc, and pressure plate).

\section{Fundamental Principles}

The main system of the friction clutch consists of pressure plate, clutch disc, and flywheel as shown in Figure 1. When the clutch starts to engage, the slipping will occur between contact surfaces due to the difference in the velocities between them (slipping period), and after this period all contact parts are rotating at the same velocity without slipping (full engagement period). A high amount of the kinetic energy converted into heat energy at interfaces according to the first law of thermodynamics during the slipping period and the heat generated between contact surfaces will be dissipated by conduction between friction clutch components and by convection to environment; in addition to the thermal effect due to the slipping, there is another load condition which is the pressure contact between contact surfaces. In the second period, there are three types of load conditions: the temperature distribution from the last period (slipping period), the pressure between contact surfaces due to the axial force of diaphragm spring, and the centrifugal force due to the rotation of the contact parts. Figure 2 shows the load conditions during the engagement cycle of the clutch, where $t_{s}$ is the slipping time and $T$ is the transmitted torque by clutch.

\section{Finite Element Formulation}

This section presented the steps to simulate the contact elements of friction clutch using ANSYS software. Moreover, it gives more details about the types of contacts and algorithms which are used in this software.

The first step in this analysis is the modelling; due to the symmetry in the geometry (frictional lining without grooves) and boundary conditions of the friction clutch (taking into consideration the effect of the pressure and centrifugal force loads, and neglected the effect of thermal load due to the slipping), three-dimensional FEM model (wedge $/ 15^{\circ}$ ) can be used to represent the contact between the clutch elements during the steady-state period as shown in Figure 3.

There are three basic types of contacts used in ANSYS software: single contact, node-to-surface contact, and surface-to-surface contact. Surface-to-surface contact is the most common type of contact used for bodies that have arbitrary shapes with relative large contact areas. This type of contact is most efficient for bodies that experience large values of relative sliding such as block sliding on plane or sphere sliding within groove [7]. Surface-to-surface contact is the type of contact assumed in this analysis because of the large areas of clutch elements in contact.

In this work, it has been assumed two types of load conditions affect on the clutch system during the steadystate period (full engagement period) the contact pressure between clutch elements due to the axial force by diaphragm spring and the centrifugal force due to the rotation.

The elements used for contact model are as follows.

(i) "Solid186": the element is defined by 20 nodes having three degrees of freedom per node: translations in the nodal $x$-, $y$-, and $z$-directions (used for all elements 


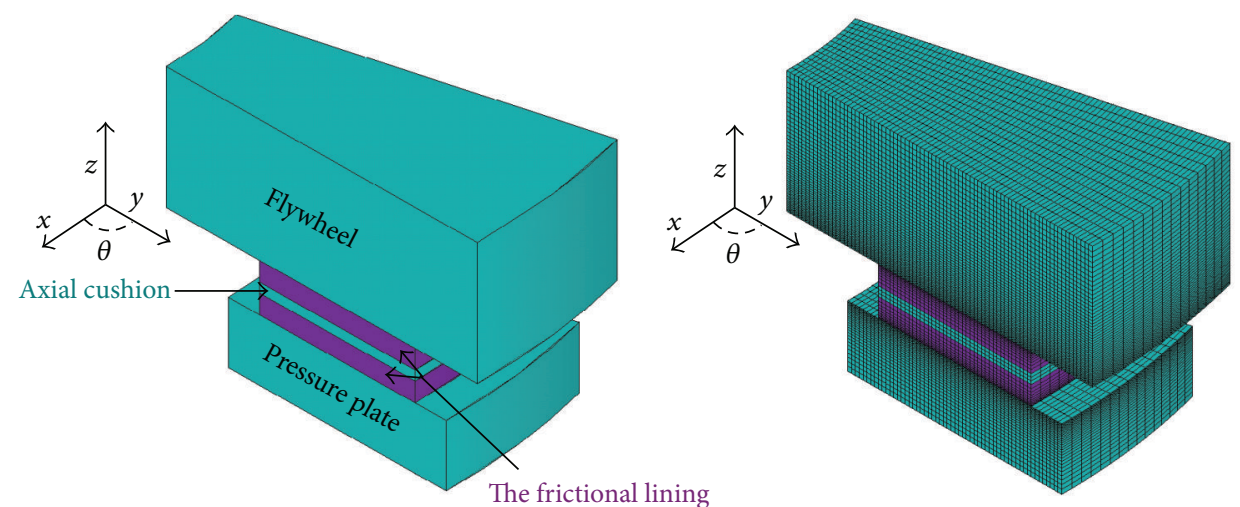

FIgURE 3: The contact and FEM models for clutch system (number of elements $=19464$ ).

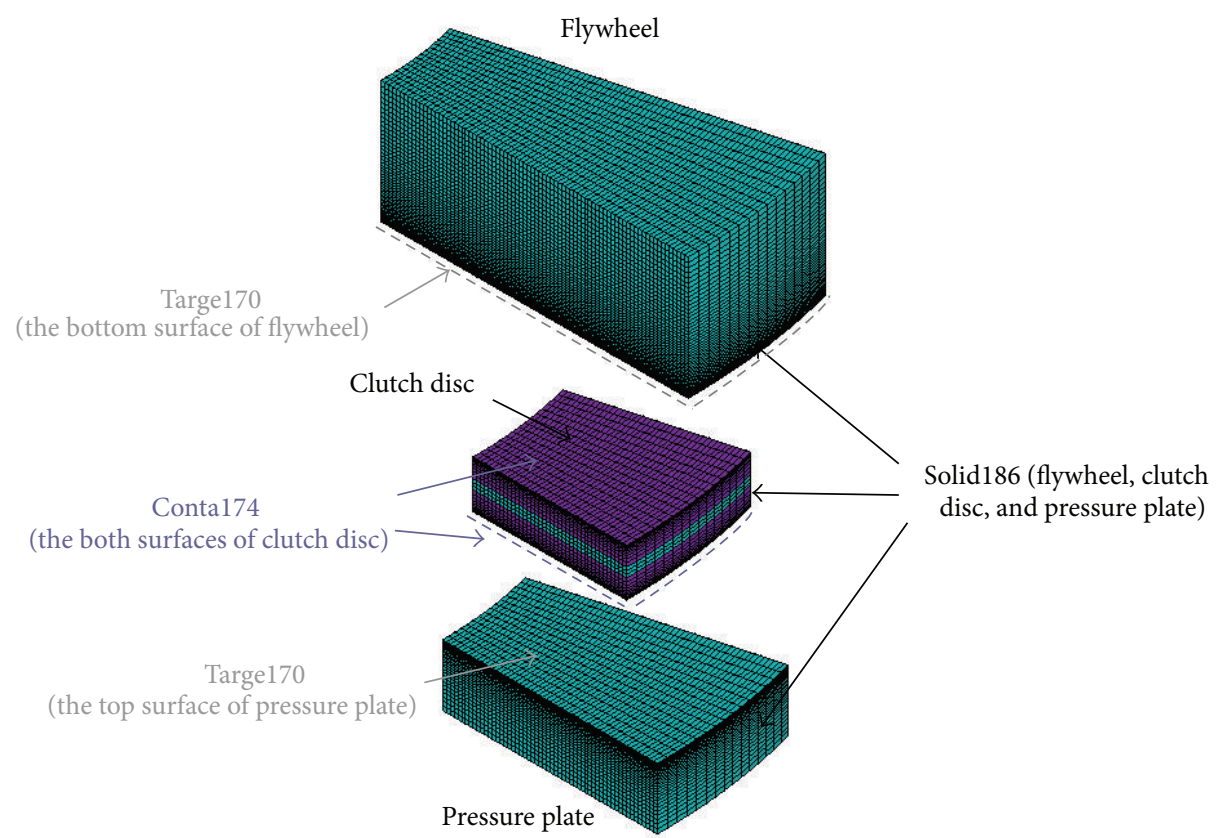

FigURE 4: Schematic elements used for the friction clutch system.

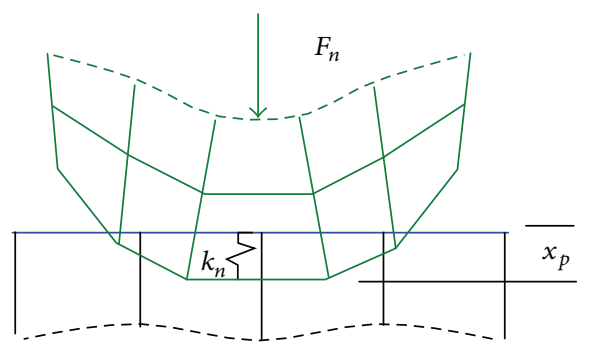

FIGURE 5: The contact stiffness between two contact bodies.

of the clutch parts (flywheel, clutch disc, and pressure plate)).

(ii) "Conta174": the element is used to represent contact and sliding between $3 \mathrm{D}$ surfaces. This element has three degrees of freedom at each node: translations in the nodal $x$-, $y$-, and $z$-directions (used for contact surfaces that are the upper and lower surfaces of clutch disc).

(iii) "Targel70": the element is used to represent various 3D "target" surfaces for the associated contact elements. The contact elements themselves overlay the solid, shell, or line elements describing the boundary of a deformable body and are potentially in contact with the target surface (used for the target surfaces that are the lower surface of the flywheel and the upper surface of the pressure plate).

Figure 4 shows the details about schematic for all elements which has been used in this analysis. 


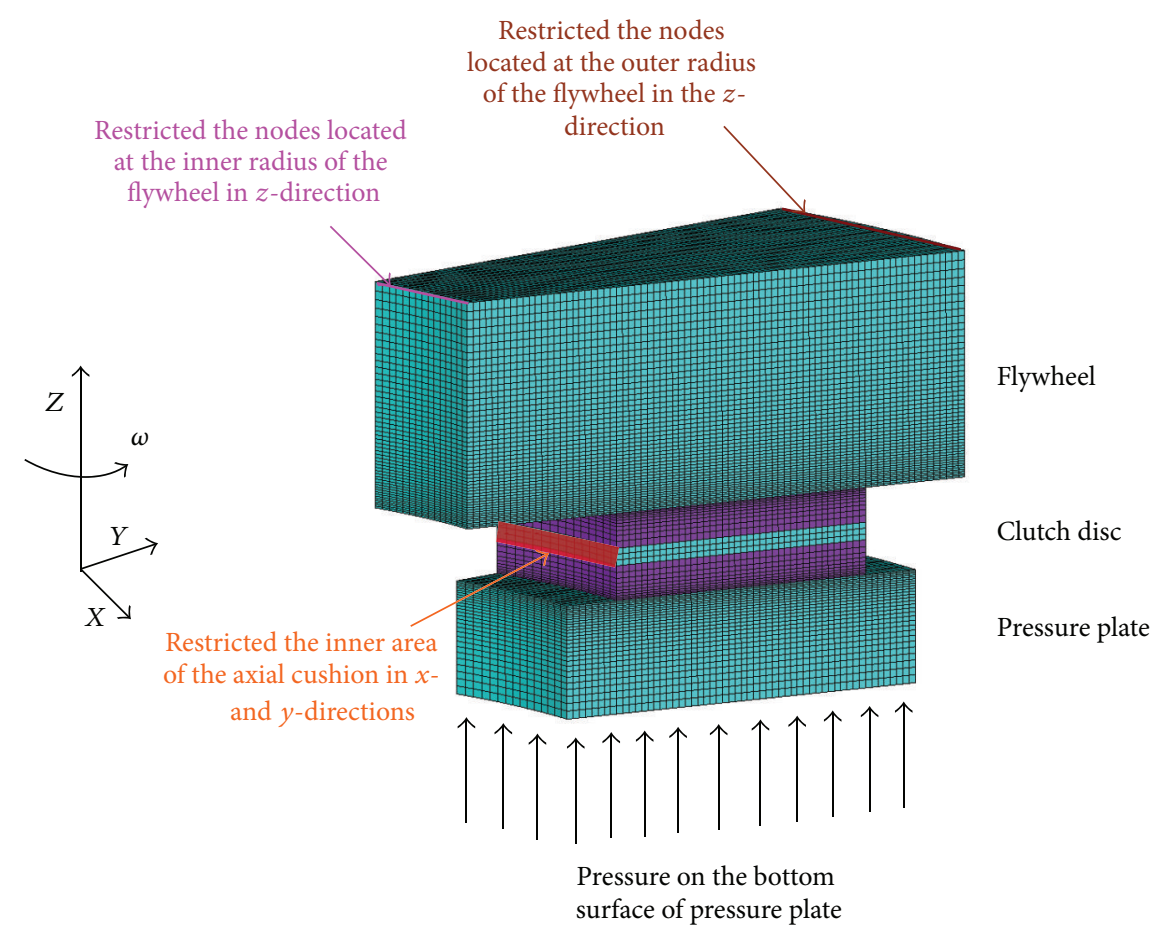

FIGURE 6: FE models with the boundary conditions.

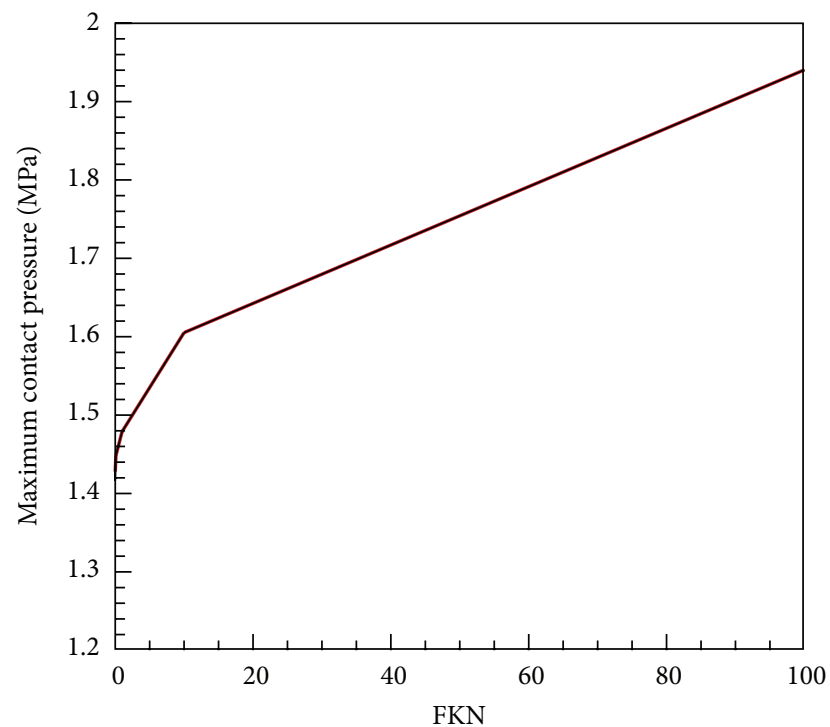

- Augmented Lagrange method

— Penalty method

FIGURE 7: The variation of the maximum contact pressure for clutch system.

The stiffness relationship between contact and target surfaces will decide the amount of the penetration. Higher values of contact stiffness will decrease the amount of penetration but can lead to ill conditioning of the global stiffness matrix and convergence difficulties. Lower values of contact stiffness can lead to certain amount of penetration and low enough to

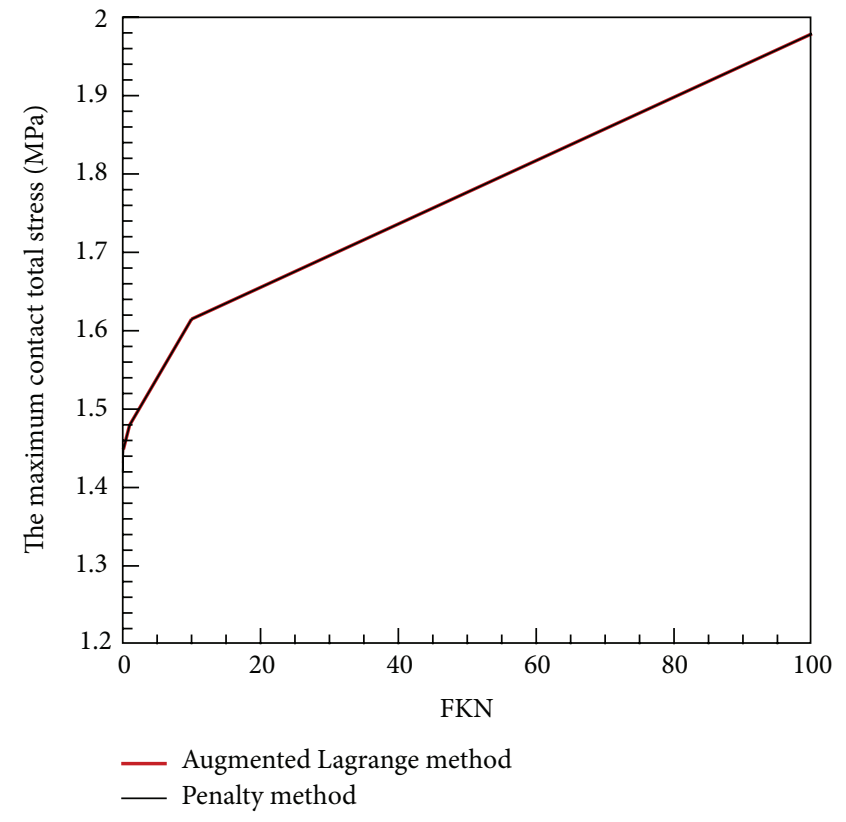

FIGURE 8: The variation of the maximum contact total stress for clutch system.

facilitate convergence of the solution. The contact stiffness for an element of area $A$ is calculated using the following formula [8]:

$$
F_{k n}=\int\left\{f_{i}\right\}(e)\left\{f_{i}\right\}^{T} d A
$$




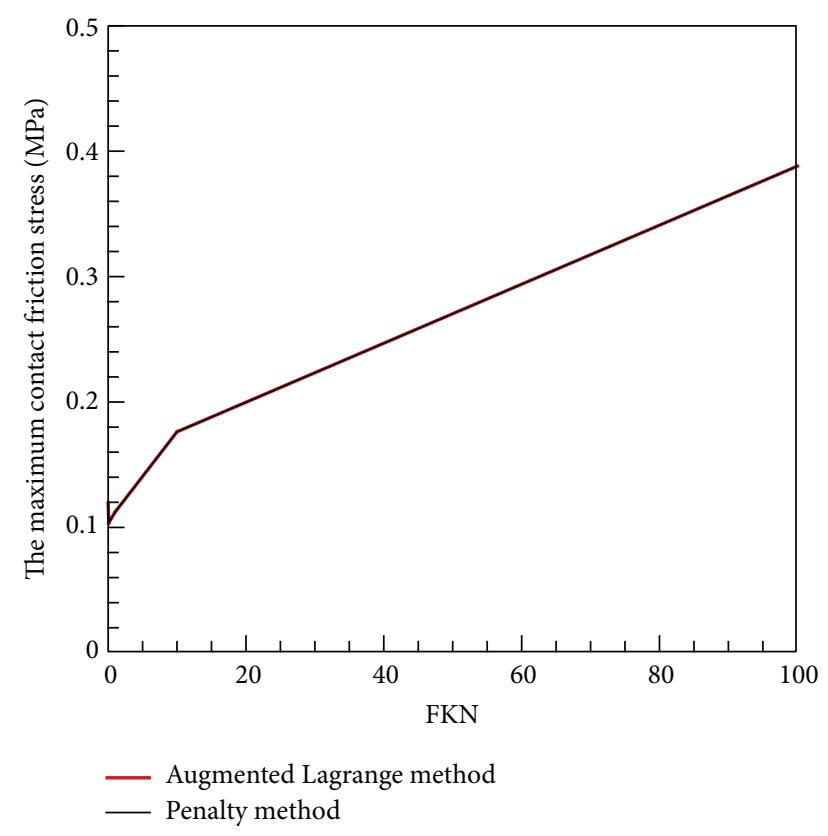

FIGURE 9: The variation of the maximum contact friction stress for clutch system.

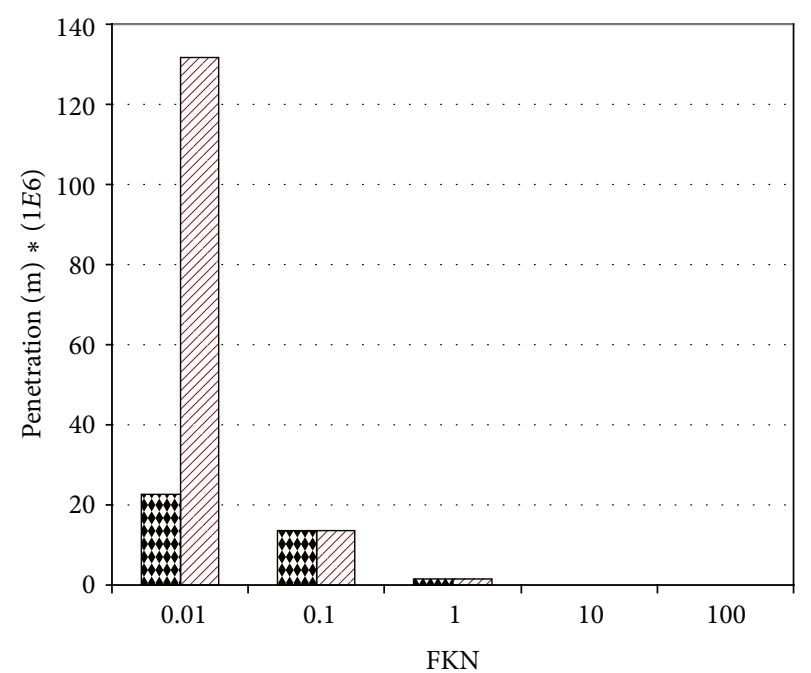

Augmented Lagrange method

$\square$ Penalty method

FIGURE 10: The variation of the maximum contact penetration for clutch system.

The default value of the contact stiffness factor (FKN) is 1 , and it is appropriate for bulk deformation. If bending deformation dominates the solution, a smaller value of FKN $=0.1$ is recommended. There are five algorithms used for surface-to-surface contact type which are as follows.

(i) Penalty method: this algorithm used constant "spring" to establish the relationship between the two contact surfaces (Figure 5). The contact force (pressure) between two contact bodies can be written as follows:

$$
F_{n}=k_{n} x_{p}
$$

where $F_{n}$ is the contact force, $k_{n}$ is the contact stiffness, and $x_{p}$ is the distance between two existing nodes or separate contact bodies (penetration or gap).

(ii) Augmented Lagrange (default): this algorithm is an iterative penalty method. The constant traction (pressure and frictional stresses) is augmented during equilibrium iterations so that the final penetration is smaller than the allowable tolerance. This method usually leads to better conditioning and is less sensitive to the magnitude of the constant stiffness. The contact force (pressure) between two contact bodies is

$$
F_{n}=k_{n} x_{p}+\lambda,
$$

where $\lambda$ is the Lagrange multiplier component.

(iii) Lagrange multiplier on contact normal and penalty on tangent: this method was applied on the constant normal and penalty method (tangential contact stiffness) on the frictional plane. This method enforces zero penetration and allows small amount of slip for the sticking contact condition. It requires chattering control parameters as well as the maximum allowable elastic slip parameter.

(iv) Pure Lagrange multiplier on contact normal and tangent: this method enforces zero penetration when contact is closed and "zero slip" when sticking contact occurs. This algorithm does not require contact stiffness. Instead it requires chattering control parameters. This method adds contact traction to the model as additional degrees of freedom and requires additional iterations to the stabilized contact conditions. It often increases the computational cost compared to the augmented Lagrangian method.

(v) Internal multipoint constraint: this method is used in conjunction with bonded contact and no separation contact to model several types of contact assemblies and kinematic constraints.

Three-dimensional finite element model of the friction clutch system with boundary conditions is shown in Figure 6 . A mesh sensitivity study was done to choose the optimum mesh from computational accuracy point of view. The full Newton-Raphson method with unsymmetric matrices of elements is used in this analysis assuming a large-deflection effect. In all computations for the friction clutch model, a homogeneous and isotropic material has been assumed and all parameters and materials properties are listed in Table 1. In this analysis also it is assumed that there are no cracks in the contact surfaces and the actual contact area is equal to the nominal contact area. 

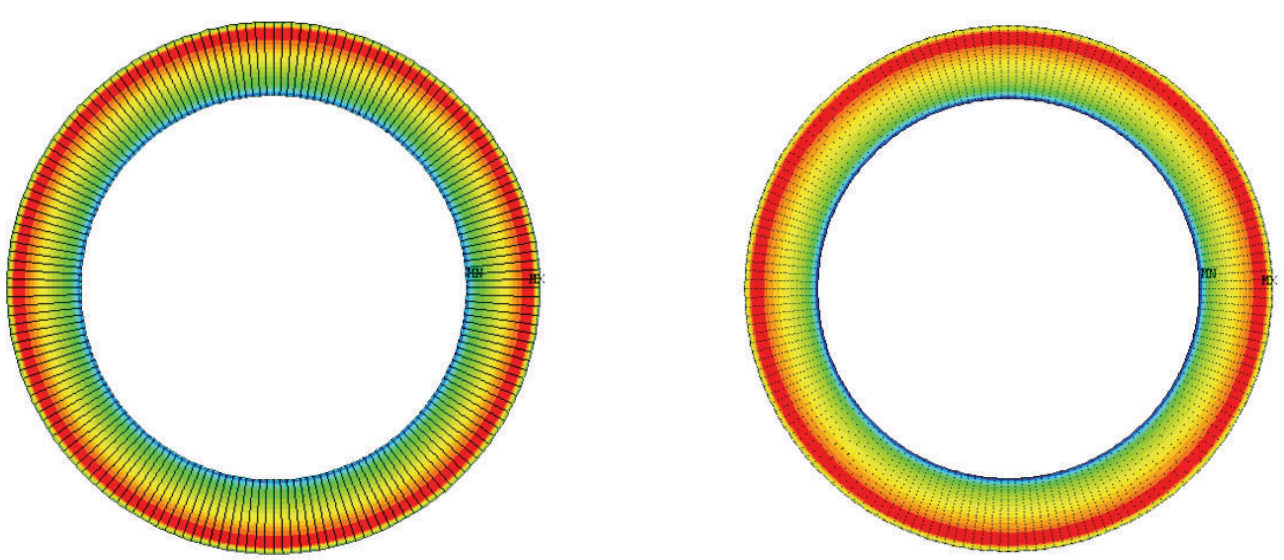

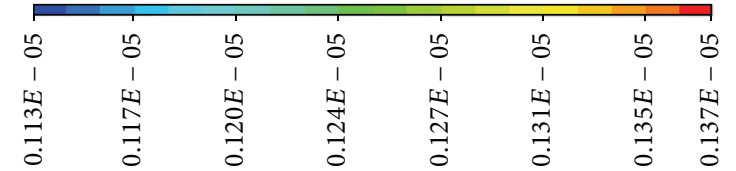

(a) Clutch disc surface/flywheel side

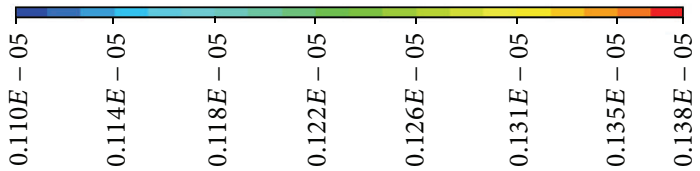

(b) Clutch disc surface/pressure plate side

FIGURE 11: The distribution of the contact penetration $(\mathrm{m})$ of clutch disc $(\mathrm{FKN}=1)$.

\section{Results and Discussions}

Series of computations have been carried out using ANSYS13 software to study the contact pressure, penetration, and stresses between contact surfaces of clutch (pressure plate, clutch disc, and flywheel) during a full engagement period using different algorithms and contact stiffness factor values.

The variations of the maximum contact pressure and contact total stress with FKN for clutch system (flywheel, clutch disc, and pressure plate) using penalty and augmented Lagrange algorithms are shown in Figures 7 and 8. From these figures, it can be seen that the identical results when using penalty and Lagrange augmented (default) methods for $\mathrm{FKN} \geq 1$. The maximum total contact stresses have the same behaviour of the contact pressure when using the same values of FKN. The maximum contact pressure value in the contact surfaces of clutch disc is found to be $1.94 \mathrm{MPa}$ when $\mathrm{FKN}=10$. The percentage increase in maximum contact pressure when FKN changes from 0.01 to 100 is found to be $35.8 \%$ and $37 \%$ corresponding to penalty and augmented Lagrange methods, respectively.

Figure 9 shows the variation of maximum contact friction stress of clutch disc with FKN using different algorithms. It can be seen that the total contact stresses have the same behavior for the maximum contact pressure, but the range of values of friction stresses is lower than that of the contact stresses due to very small slipping happened during this analysis.

The variation of the penetration with FKN using different algorithms for the clutch disc is shown in Figure 10. It can be noted for both cases (the penalty and augmented methods) that the values of penetration decrease when FKN increases. The ratio of the maximum penetration $\mathrm{Pe}_{\max }\left(\mathrm{Pe}_{\max }=\right.$ the maximum penetration using penalty method/the maximum penetration using augmented Lagrange method) at FKN $=0.01$ is found to be 5.8, and $\mathrm{Pe}_{\max }$ is found to be 1 (approximately) for $\mathrm{FKN} \geq 1$.

The distribution state of penetration for both sides of clutch disc (flywheel side and pressure plate side) is as shown in Figure 11. From this figure, it can be seen that the maximum values of penetration for both sides of clutch disc occur near the outer disc radius and the minimum values occur at inner radius. The maximum value of the penetration at the pressure plate side is higher than at the flywheel side.

Figure 12 shows the contour of the contact pressure distribution of friction clutch disc surfaces. It can be seen that the contact pressure grows approximately linearly from minimum value number at inner radius to maximum value number near the outer radius for both sides of clutch disc.

Figure 13 illustrates the contour of the contact friction stress of both surfaces of clutch disc. It can be noted that the higher value of contact friction stress occurs at the inner and outer radii and the lower value occurs near the mean radius of clutch disc for both surfaces.

\section{Conclusions and Remarks}

The variations of the contact pressure, penetration, total contact stress, and contact friction stress of the friction clutch using different contact algorithms and different values of FKN are investigated. Three-dimensional finite element model for the contact elements of clutch was conducted to obtain the numerical results.

The present work presents a simplified model of clutch to determine the contact pressure between contact surfaces during a full engagement period.

The conclusions obtained from the present analysis are summarized as follows. 

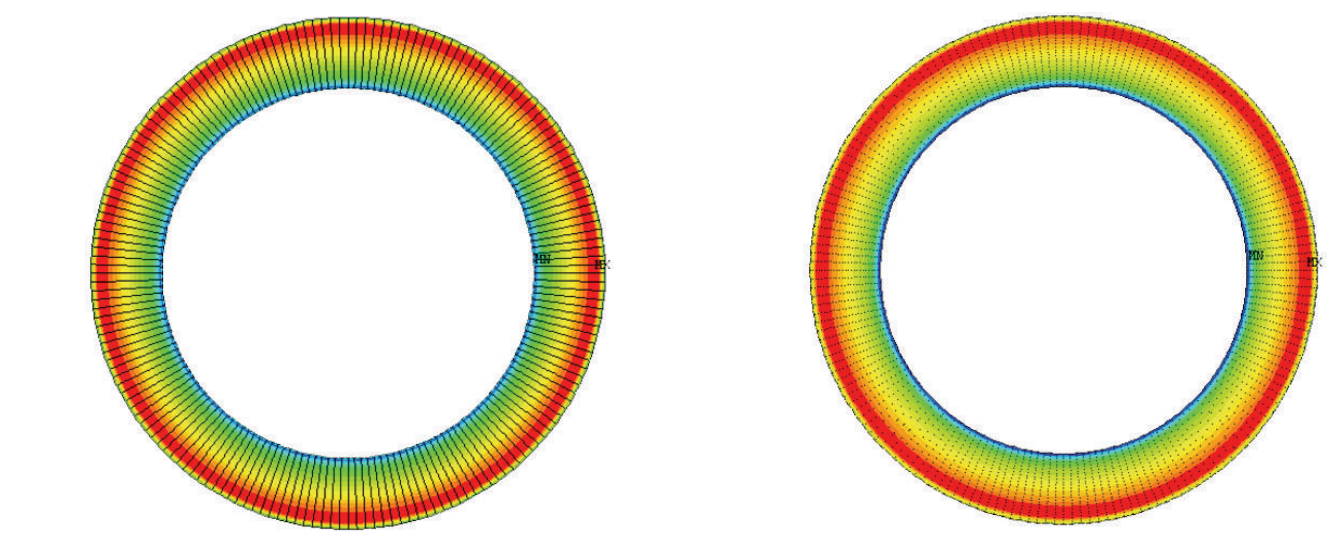

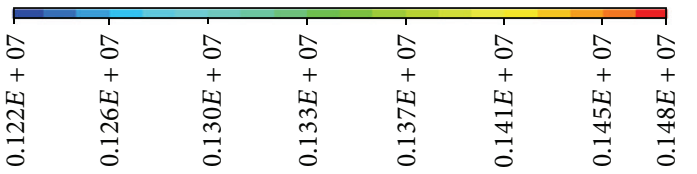

(a) Clutch disc surface/flywheel side

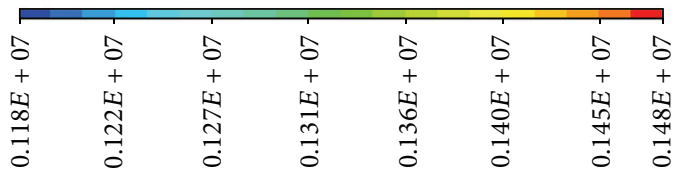

(b) Clutch disc surface/pressure plate side

FIGURE 12: The distribution of the contact pressure $(\mathrm{MPa})$ of clutch disc $(\mathrm{FKN}=1)$.

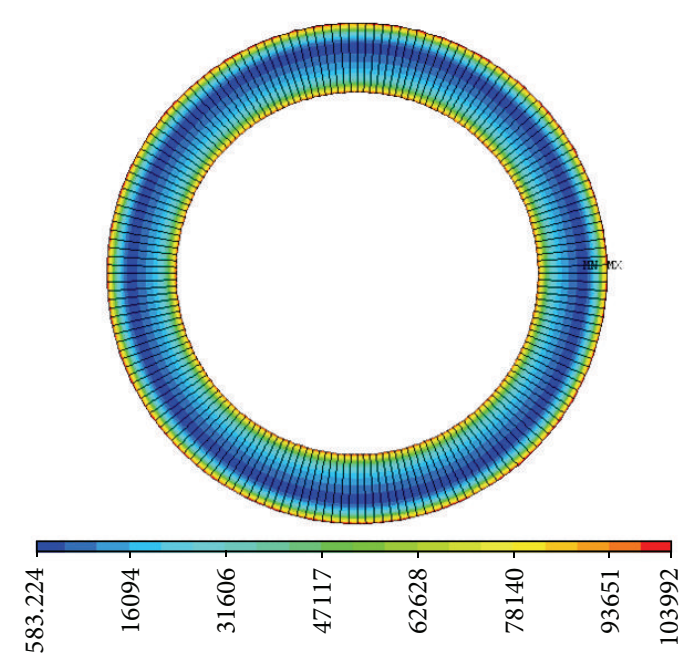

(a) Clutch disc surface/flywheel side

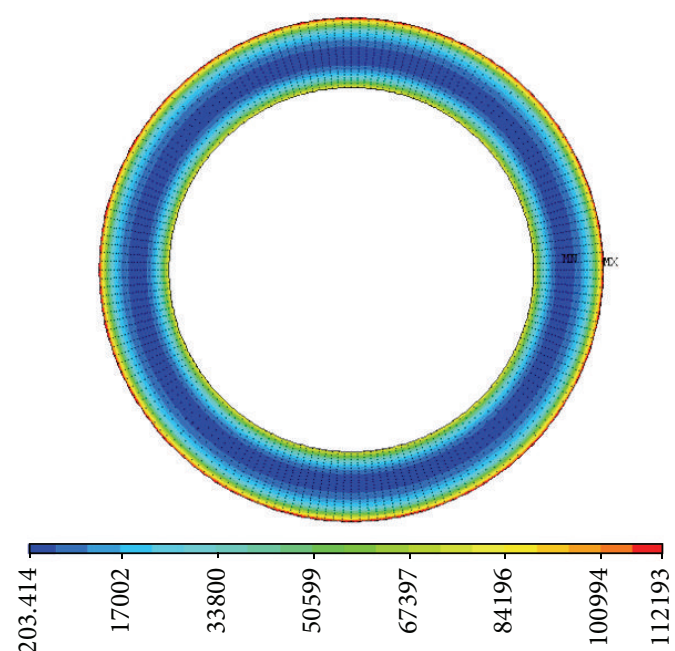

(b) Clutch disc surface/pressure plate side

FIGURE 13: The distribution of the contact friction stress $(\mathrm{MPa})$ of clutch disc $(\mathrm{FKN}=1)$.

(1) The value of FKN is very important and has effect on the values of contact pressure, and the contact pressure is directly proportional to FKN for both contact methods (penalty and augmented).

(2) The penalty method has sensitivity for FKN more than the augmented method for FKN $<1$.

(3) The maximum and minimum values of contact pressure and penetration occur near the outer disc radius and at the inner disc radius, respectively.

(4) Very small value of slip occurs between the contact surfaces due to the high contact pressure between clutch elements, and this slip leads to the generation of contact friction stresses. The maximum values of contact friction stresses occur at the inner and outer disc radii, and the minimum value occurs near the mean radius of clutch disc.

The permanent deformations and thermal cracks on the contact surfaces of clutch if taken into consideration will affect the contact pressure distribution and the actual contact area will change. These disadvantages will focus the contact pressure on small region compared with the nominal contact area.

\section{References}

[1] A. M. Al-Shabibi and J. R. Barber, "Transient solution of the unperturbed thermoelastic contact problem," Journal of Thermal Stresses, vol. 32, no. 3, pp. 226-243, 2009. 
[2] Y. L. Choon, C. Ilsup, and S. C. Young, "12Finite element analysis of an automobile clutch system," Key Engineering Materials, vol. 353-358, no. 4, pp. 2707-2711, 2007.

[3] M. M. Shahzamanian, B. B. Sahari, M. Bayat, Z. N. Ismarrubie, and F. Mustapha, "Transient and thermal contact analysis for the elastic behavior of functionally graded brake disks due to mechanical and thermal loads," Materials and Design, vol. 31, no. 10, pp. 4655-4665, 2010.

[4] M. El-Sherbiny and T. P. Newcomb, “Temperature distributions in automotive dry clutches," Proceedings of the Institution of Mechanical Engineers, vol. 190, no. 34, pp. 359-365, 1976.

[5] E. A. Al-Bahkali and J. R. Barber, "Nonlinear steady state solution for a thermoelastic sliding system using finite element method," Journal of Thermal Stresses, vol. 29, no. 2, pp. 153-168, 2006.

[6] A. A. Yevtushenko, E. G. Ivanyk, and O. O. Yevtushenko, "Exact formulae for determination of the mean temperature and wear during braking," Warme- und Stoffubertragung Zeitschrift, vol. 35, no. 2, pp. 163-169, 1999.

[7] ANSYS Contact Technology Guide, ANSYS Release 11. 0 Documentation, ANSYS, Inc.

[8] G. A. Mohr, "Contact stiffness matrix for finite element problems involving external elastic restraint," Computers and Structures, vol. 12, no. 2, pp. 189-191, 1980. 

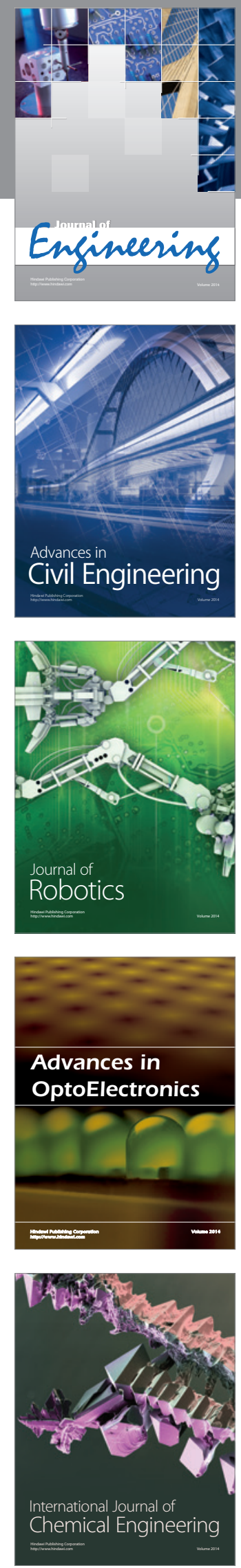

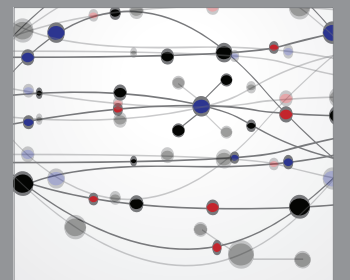

The Scientific World Journal
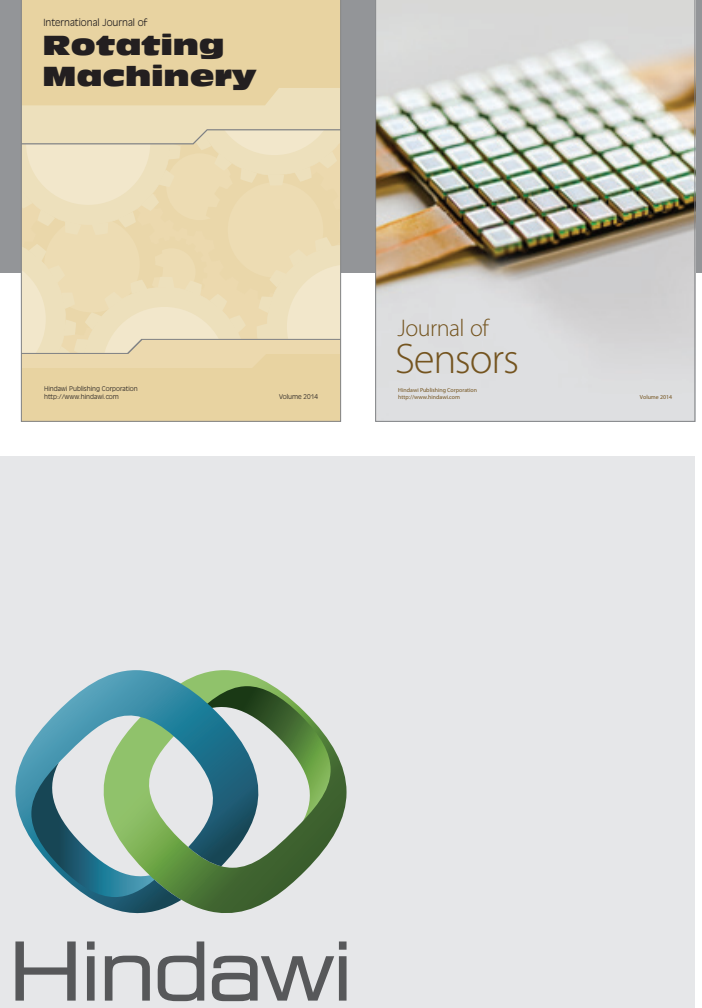

Submit your manuscripts at http://www.hindawi.com
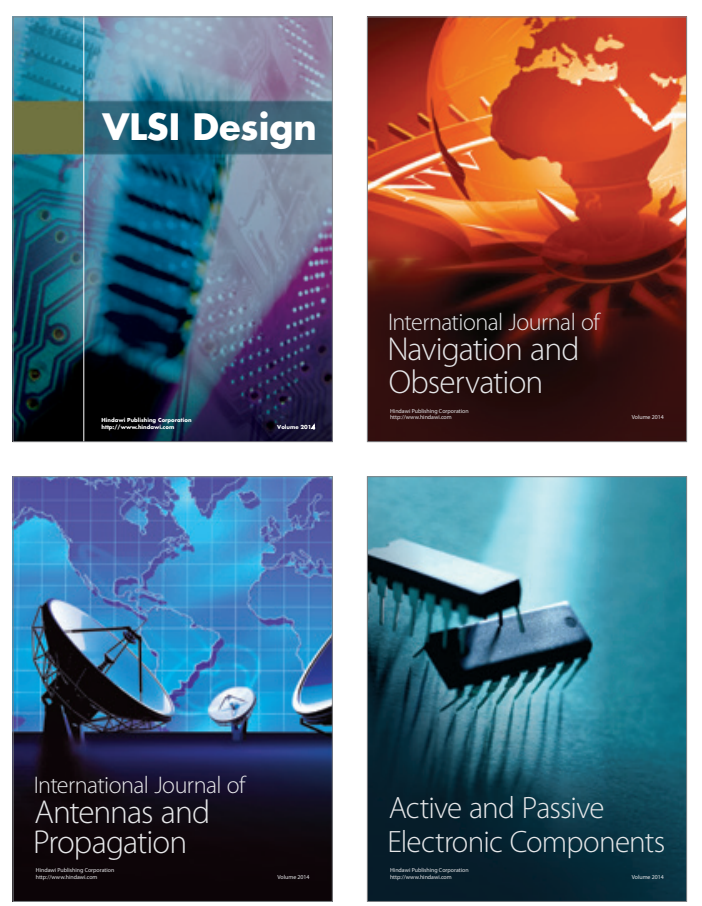
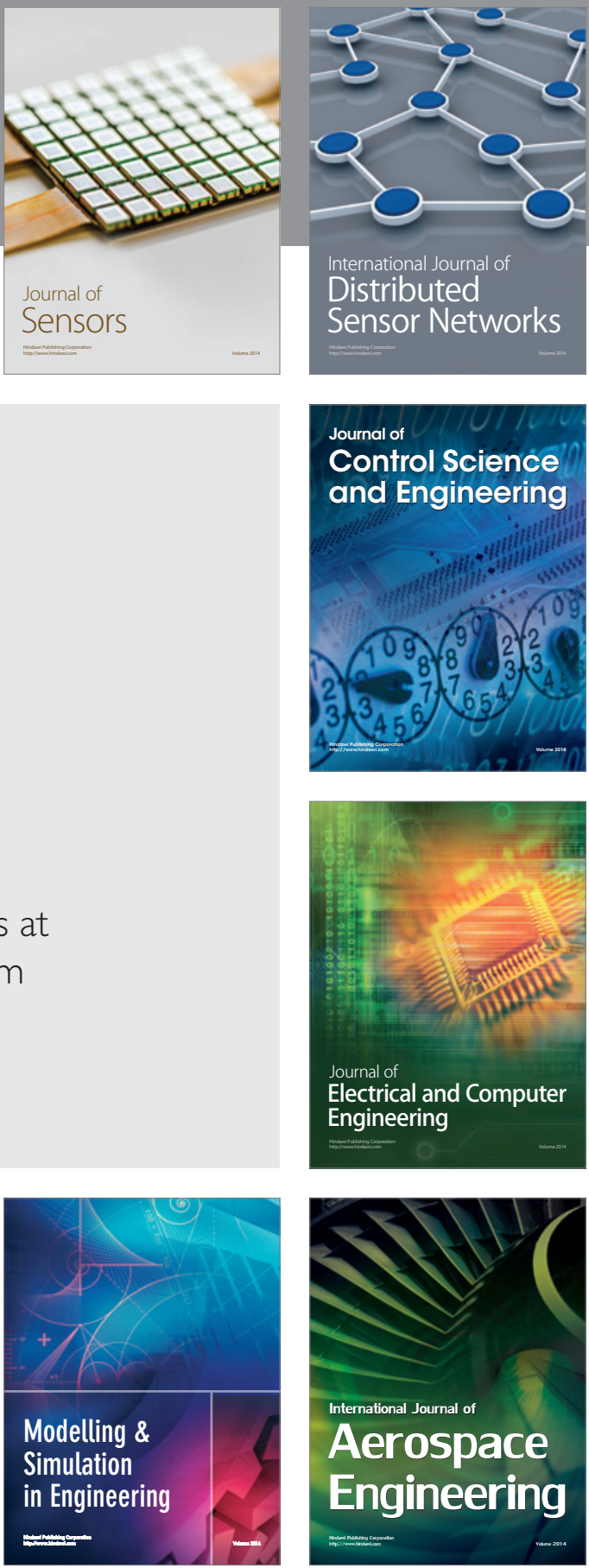

Journal of

Control Science

and Engineering
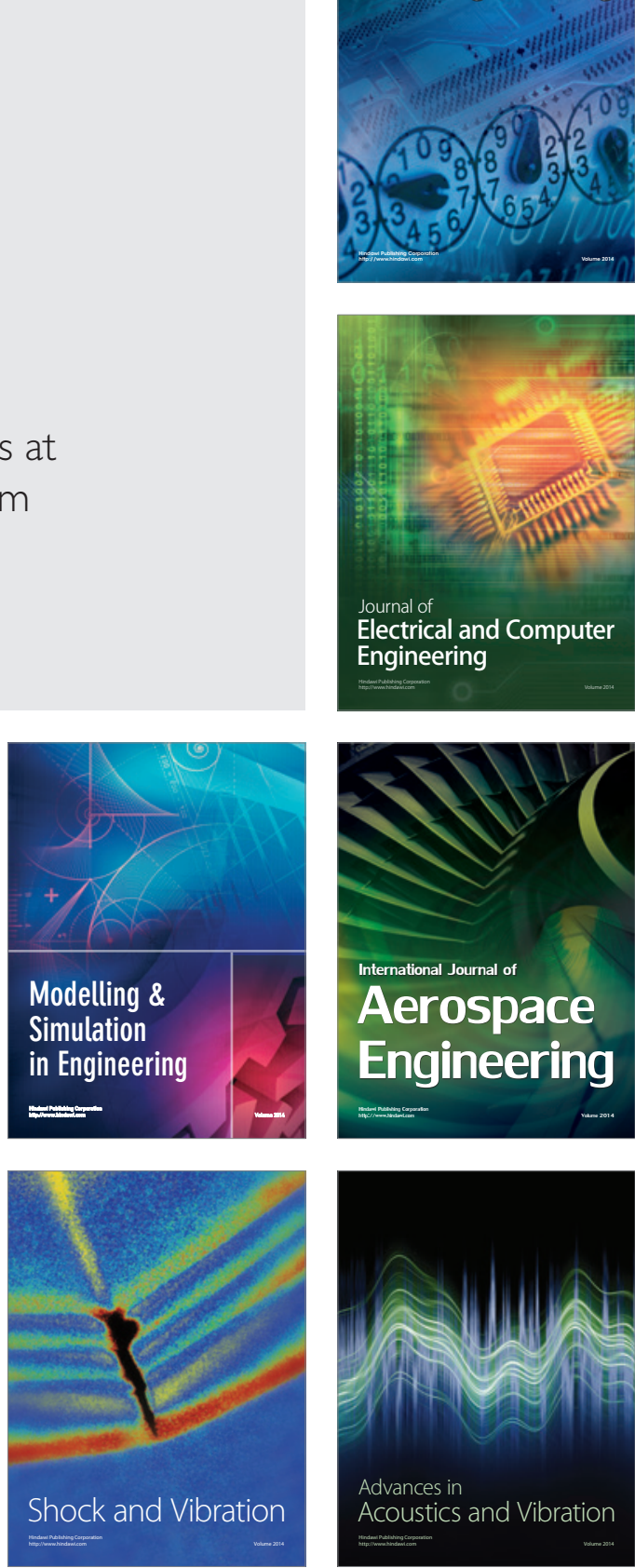